\title{
Morphological, physiological, and biochemical responses of onion (Allium cepa L.) breeding lines to single and combined salt and drought stresses
}

Zahide Neslihan ÖZTÜRK GÖKÇE ( $\nabla$ zn_ozturk@hotmail.com )

Nigde University: Nigde Omer Halisdemir Universitesi https://orcid.org/0000-0002-5077-5141

\section{Ali Fuat Gokce}

Nigde University: Nigde Omer Halisdemir Universitesi

Muhammad Daniyal Junaid

Nigde University: Nigde Omer Halisdemir Universitesi

Usman Khalid Chaudhry

Nigde University: Nigde Omer Halisdemir Universitesi

\section{Research Article}

Keywords: Abiotic stress, combined salt and drought stress, onion genotypes, yield, biochemical, PCA

Posted Date: November 29th, 2021

DOI: https://doi.org/10.21203/rs.3.rs-1113185/v1

License: (a) (i) This work is licensed under a Creative Commons Attribution 4.0 International License.

Read Full License

Version of Record: A version of this preprint was published at Euphytica on February 12th, 2022. See the published version at https://doi.org/10.1007/s10681-022-02980-7. 


\section{Abstract}

Abiotic stresses deteriorate plant growth resulting in devastating yield losses. Salt stress solely cause ionic toxicity and disturbed homeostasis, whereas combined salt and drought stress has more pronounced effects on plants. Here we performed a pot experiment using 32 Turkish onion breeding lines and commercial cultivars for screening against salt, drought and combined salt and drought stress treatments initiated at the bulbification stage under greenhouse conditions. Physiological characteristics, such as gaseous exchange traits, chlorophyll index, leaf temperature, and morphological traits including number of leaves, length and diameter of leaf were measured during stress treatment and yield response of the onions was quantified by measuring bulb length, bulb diameter and bulb weight after harvest. Proline and malondialdehyde accumulation were estimated for biochemical effect of stress on onion genotypes. All genotypes responded differentially to the applied single and combined stresses. Overall results revealed that the breeding lines K25, U6, U17 and commercial cultivar K58, were grouped as sensitive across all stresses, whereas the breeding lines K41, U47, U49 and commercial cultivar K52, were found to be the most stable upon stress. These resilient genotypes can be used as breeding material for future abiotic stress studies.

\section{Introduction}

Onion is a cross-pollinated, biennial crop which belongs to Alliaceae family (Ghodke et al. 2018). It is a robust crop which can grow under a wide range of climatic conditions, but its full potential can be observed under mild climatic conditions (Mubarak and Hamdan 2018). This bulbous vegetable is grown and consumed globally (Havey and Ghavami 2018). Depending on the area of cultivation, onion is considered as biannual or perennial vegetable. It originally domesticated from the region including SouthWestern Pakistan, Iran, and Afghanistan (Brewster, 1994). Among vegetables, onion ranks second as the most cultivable vegetable crop with production of 97.8 million tons. Turkey ranks 6 th in the world with the total production of 2.13 million tons (FAOSTAT 2019).

Agricultural development has been seriously hindered by abiotic stresses in recent decades. Major abiotic stresses include drought, heat, cold and salt stress (Asim et al. 2021; Gürel et al 2016). These abiotic stresses alone or in combination pose threat to agricultural yield and quality, moreover it is estimated that $50 \%$ of major crop yield is affected by these mentioned abiotic stresses (Singh et al. 2018). Abiotic stresses disturb normal plant functioning by limiting photosynthetic activity, which in turn causes reduced plant yield (Clay et al. 2014). Excessive amounts of undesirable salts in soil like $\mathrm{Na}^{+}$and $\mathrm{Cl}^{-}$, are taken up by plant roots, and cause deterioration in plant functioning and growth (Chaudhry et al. 2021). It is also known that high salt concentration affects water uptake by plant due to presence of salts around plant roots which also leads to oxidative stress (Golldack et al. 2014). Under drought, plants are unable to up take adequate moisture for their growth affecting physio-biochemical phenomenon in plants (Junaid et al. 2021). Furthermore, morphological, physiological, and biochemical functioning of plants are interrupted with onset of drought and salinity. Extent of damage of these stresses on every crop varies depending on the nature of plant species (Kinraide 1999). 
Allium cepa $\mathrm{L}$. is a shallow rooted crop its root penetration is around $0.18 \mathrm{~m}$ which means it cannot uptake moisture from deep soils (Drinkwater and Janes 1957). Onion root texture makes it highly sensitive to drought stress when it is grown in coarse soils (Rao et al. 2016). Previous studies indicated that water deficit negatively affects onion bulb formation which resultantly hinders its quality and yield (Chaudhry et al. 2020; Ghodke et al. 2020). On the other hand, salinity especially in arid regions derange plant metabolic system by excessive accumulation of inorganic salts in plants (Shahzad et al. 2017). There is still limited literature available on the association of drought and salinity with physiobiochemical mechanisms in onion.

This study aimed to explore the effects of drought, salinity, and combined stresses on onion to screen tolerant and susceptible onion genotypes that can used in further breeding studies. Morpho-physiological and biochemical changes were considered to screen short day and long day onion breeding lines. According to our knowledge, this is the first study evaluating the response of onion to combined salt+drought stress. It was hypothesized that based on the results obtained 32 short and long-day onion breeding lines all the observed physio-biochemical attributes can be used as a criterion for the rapid selection with a higher tolerance to applied stresses.

\section{Material And Methods 2.1 Plant material}

A total of 32 yellow, white, and red scale colored 12 short-day onion genotypes including 8 advanced breeding lines $\mathrm{K} 18, \mathrm{~K} 20, \mathrm{~K} 25, \mathrm{~K} 28, \mathrm{~K} 35, \mathrm{~K} 37, \mathrm{~K} 39$, K41, and 4 commercial cultivars $\mathrm{K} 51, \mathrm{~K} 52, \mathrm{~K} 58$ and $\mathrm{K} 59$ were used. Likewise, 20 long-day includes 16 advanced breeding lines U2, U6, U9, U10, U11, U12, U16, U17, U23, U24, U29, U31, U33, U44, U47, U49, and 4 commercial cultivars U-55, U-57, U-63 and U-65 were used in this study. Short-day onions were sown during November whereas, long-day onions were sown during December 2019 in semi controlled (no artificial lightening, temperature control with $20^{\circ} \mathrm{C}$ day $/ 10^{\circ} \mathrm{C}$ night until bulb formation, $25^{\circ} \mathrm{C}$ day $/ 15^{\circ} \mathrm{C}$ night during stress treatment, ambient humidity with 40 to $70 \%$ during the growing season) greenhouse of Niğde Ömer Halisdemir University, Ayhan Şahenk Faculty of Agricultural Sciences and Technologies, Agricultural Genetic Engineering. Total 10 seeds of every genotype were planted in 10-liter pots with 3: 1 ratio of peat and perlite. A total of 12 (384) pots were planted for each breeding line, 3 for control (C), 3 for salt stress (SS) application, 3 for drought stress (DS) application, and 3 for salt and dry stress (DSS) application. At all plant growth stages, recommended plant protection procedures were performed to combat weeds, pests and diseases.

\subsection{Stress treatment}

It was initiated at onion bulb formation stage, Salt stress treatment was carried out as described in our earlier study Chaudhry et al. (2020), as 3 pots for each onion line for short-day onions were used, considering the water requirement of the soil in the pots, the concentrations of salt was applied with 3 days interval with the salt concentrations of $75 \mathrm{mM}, 100 \mathrm{mM}, 125 \mathrm{mM}$, and three times $150 \mathrm{mM}$ saline water was applied. The application of salt and drought stress was carried out by applying limited 
irrigation mixed with salt concentration $(\mathrm{NaCl})$ at the rate of $70 \%$ of the soil saturation capacity. The application of salt and drought stress was continued for 20 days. Combined salt and drought stress was applied by mixing the same amounts of salt used for salinity stress in each combined stress treatment pot. The drought stress application was carried out by stopping irrigation in onion plants belonging to drought stress group at bulb formation stage. The stress application continued for 20 days in the shortand long-day onions (Supplementary Fig. 1). After application of stress, drought stressed plants were well- watered, and salinity stressed plants were washed with normal irrigation water to drain excessive salt. Afterwards, plants were allowed to grow until they showed bending of the neck for harvest of onion bulbs. For biochemical sample collection leaf samples were collected from 3rd or 4th fresh leaves in 3 replications for further biochemical analysis.

\subsection{Physiological measurements}

Physiological traits were measured from all four treatment groups (control, salt stress, salt+drought stress, and drought stress) by selecting three random plants from each group. Measurements were taken in triplicates from 3 rd or 4 th leaf of the plants which were properly exposed to the light.

\subsection{Gaseous exchange traits}

Photosynthetic rate, transpiration rate, and stomatal conductance measurements from all treatment groups were taken by constant light intensity of photosynthesis device $\left(1500 \mu \mathrm{mol} \mathrm{m}{ }^{-2} \mathrm{sec}^{-1}\right), \mathrm{CO}_{2}$ amount $(400 \mu \mathrm{mol})$ and airflow $\left(500 \mu \mathrm{mol} \mathrm{s}^{-1}\right)$ and when photosynthesis gained steady state, the measurement was recorded using a portable gas exchange system LiCor 6400 XT (Li-Cor Biosciences, USA). All measurements were taken in three replicates and mean values were used for data analysis.

\subsection{Chlorophyll index}

Leaf chlorophyll index was measured in three replicates by using SPAD 502 Chlorophyll-Meter (Soil Plant Analysis Development; Minolta, Japan).

\subsection{Leaf temperature}

Leaf temperature was also measured in three replicates from each pot by using Infrared Thermometer (IRT) device (MASTECH BM380).

\subsection{Morphological and yield traits}

It was measured by selecting three plants from each pot. Length of leaf $(\mathrm{cm})$ and diameter of leaf $(\mathrm{cm})$ was measured by measuring tape, whereas number of leaves was counted from each pot. Onion bulbs were harvested to measure bulb diameter $(\mathrm{cm})$, bulb length $(\mathrm{cm})$, and weight of the bulbs $(\mathrm{g})$ was measured by precise weigh balance. The leafy green part of onion plants was collected, and fresh weight was measured. After 24 hours of drying at $180^{\circ} \mathrm{C}$, samples were weighed again, and the biomass change was calculated by measuring the difference between fresh and dry weight of the leaves.

\subsection{Biochemical measurements}


Determination of proline accumulation was done based on the standard curve, created using pure proline (Chaudhry et al. 2021). Accordingly, after the completion of stress application, leaf samples were collected in liquid nitrogen from control and stress group plants. $100 \mathrm{mg}$ leaf sample was grinded in $1 \mathrm{ml}$ $75 \%$ ethanol at room temperature overnight. Then, $100 \mu$ of the upper phase of the sample was collected after centrifugation at maximum speed for 20 minutes, then transferred to a new tube and $900 \mu \mathrm{l}$ of fresh ninhydrin solution was added ( $1 \%$ ninhydrin, $60 \%$ acetic acid, $40 \%$ pure water). Then samples were kept at $100^{\circ} \mathrm{C}$ for one hour and immediately cooled at room temperature and after adding $3 \mathrm{ml}$ of toluene, they were again kept at room temperature for 24 hours. The absorbance of resulting solution was then measured by spectroscopy at $520 \mathrm{~nm}$ and amount of proline in the sample was determined with the help of the standard curve prepared using pure proline.

2-Thiobarbituric acid (TBA) test, was used to determine the amount of MDA, which is the final product in lipid peroxidation. Protocol suggested by Heath and Packer (1968) was followed, the plant sample (300 $\mathrm{mg})$ collected from the control and stress group plants were grinded in $0.1 \% 3 \mathrm{ml}(\mathrm{w} / \mathrm{v})$ trichloroacetic acid (TCA) solution. Then this sample was centrifuged at $10000 \mathrm{~g}$ for 10 minutes. $1.5 \mathrm{ml}$ of the obtained upper phase was collected; $1.5 \mathrm{ml}$ of $20 \%(\mathrm{w} / \mathrm{v})$ TCA solution containing $0.5 \%(\mathrm{w} / \mathrm{v})$ TBA was added in it. After the mixture was incubated in $90^{\circ} \mathrm{C}$ water for 20 minutes, the tubes were kept in ice to stop the reaction. Then, samples were again centrifuged at $10000 \mathrm{~g}$ for 5 minutes, the absorbance of the resultant product was measured at $532 \mathrm{~nm}$ and $600 \mathrm{~nm}$. The amount of MDA was calculated using the coefficient value of $155 \mathrm{mM}-1 \mathrm{~cm}-1$. In the absorbance measurement, $20 \%$ TCA solution containing $0.5 \%$ TBA was used.

\subsection{Statistical analyses}

Treatment data set comprised of stress environments for 32 diverse genotypes analyzed by Analysis of Variance (ANOVA). Least significant difference (LSD) was used at $\mathrm{P} \leq 0.05$ for the comparison of treatment means by Statistical package Statistix 8.1 (Tallahassee Florida, USA) (Steel et al. 1997). Pearson correlation and principal components analysis was done by using Origin 2020. The mean bulb weight of onion was subjected to analyze the genotype by environment interaction. Heritability $(\mathrm{H} 2)$ was estimated by using an equation $\mathrm{H} 2=V G / V P$, where $V G$ is genotypic variance and VP is phenotypic variance (Toker 2004). Stability analysis such as regression coefficient (bi) was done by STABILITYSOFT (Naeem and Caliskan 2020).

\section{Results}

\subsection{Analysis of variance for the observed traits}

Analysis of variance evaluated in response to salt, drought and combined salt+drought stresses showed highly significant $(p \leq 0.05)$ differences in the morpho-physiological and biochemical traits (Table 1$)$. Mean performance of all the traits depicted significant $(p \leq 0.05)$ differences that would be helpful for the selection tolerant and sensitive genotypes. The genotype and its interaction with the environment were also significant that highlighted contrasting behavior of all the tested genotypes under applied 
stresses. The genotypes subjected to specific stresses showed differential inhibition of growth suggesting that some of the genotypes were sensitive to salt stress, while others showed sensitivity to drought stress conditions.

Table 1

Two-way analysis of variance (ANOVA) of genotype, treatment and their interactions for the observed traits in onion genotypes under three treatments (salt stress, drought stress and salt+drought stress).

\begin{tabular}{|lllll|}
\hline Traits & Cultivar & Treatment & Interaction & Residual \\
\hline SPAD & 185.39 & 77.87 & 31.31 & 13.70 \\
\hline Pn & 511.65 & 1427.66 & 21.44 & 5.16 \\
\hline Gs & 0.088 & 0.089 & 0.001 & 0.00 \\
\hline E & 1.71 & 35.62 & 0.48 & 0.10 \\
\hline MDA & 39.87 & 20.68 & 5.56 & 2.77 \\
\hline PRO & 97.28 & 55.41 & 20.82 & 5.10 \\
\hline LT & 104.20 & 6.19 & 1.88 & 0.72 \\
\hline NL & 10.99 & 61.36 & 1.71 & 0.83 \\
\hline LL & 933.58 & 1863.38 & 131.89 & 48.66 \\
\hline DL & 0.62 & 2.10 & 0.15 & 0.04 \\
\hline PB & 3.10 & 47.80 & 3.00 & 1.84 \\
\hline BD & 245.27 & 590.10 & 22.65 & 7.52 \\
\hline BL & 146.15 & 467.96 & 15.87 & 5.83 \\
\hline BW & 4535.00 & 1489.98 & 5370.10 & 1532.30 \\
\hline
\end{tabular}

Numbers represent mean sum of squares at the $5 \%$ confidence level. Abbreviations: SPAD chlorophyll content, $\mathrm{Pn}$ Photosynthetic rate, $\mathrm{g}$ stomatal conductance, $\mathrm{E}$ transpiration rate, MDA malondialdehyde contents, PRO proline contents, LT leaf temperature, NL number of leaves, LL leaf length, DL leaf diameter, PB plant biomass, BD bulb diameter, BL bulb length, BW bulb weight. 
Table 2

Optimum means of bulb weight of onion and IPC1 score the genotypes tested across three stress environments.

\begin{tabular}{|c|c|c|}
\hline Genotypes & Mean bulb weight & IPC1 score \\
\hline K18 & 77.85 & 0.66 \\
\hline K20 & 73.06 & 0.48 \\
\hline K25 & 47.93 & -0.48 \\
\hline K28 & 66.81 & -0.86 \\
\hline K35 & 86.89 & -5.35 \\
\hline K37 & 84.07 & -0.54 \\
\hline K39 & 87.46 & -1.52 \\
\hline K41 & 98.15 & -0.46 \\
\hline K51 & 86.12 & 0.97 \\
\hline K52 & 96.75 & 1.69 \\
\hline K58 & 44.34 & 0.07 \\
\hline K59 & 74.22 & 2.16 \\
\hline U2 & 65.34 & -1.37 \\
\hline U6 & 41.71 & -0.33 \\
\hline U9 & 63.55 & 0.23 \\
\hline U10 & 66.92 & -0.33 \\
\hline U11 & 64.78 & -2.19 \\
\hline U12 & 97.87 & 1.44 \\
\hline U16 & 53.87 & 1.83 \\
\hline U17 & 42.76 & 0.59 \\
\hline U23 & 64.84 & 1.04 \\
\hline U24 & 63.16 & -0.73 \\
\hline U29 & 69.80 & 1.96 \\
\hline U31 & 57.02 & 2.62 \\
\hline U33 & 50.37 & 0.41 \\
\hline U44 & 65.37 & 1.37 \\
\hline
\end{tabular}




\begin{tabular}{|lll|}
\hline Genotypes & Mean bulb weight & IPC1 score \\
\hline U47 & 100.19 & 0.65 \\
U49 & 106.31 & -0.33 \\
\hline U55 & 60.82 & 0.73 \\
\hline U63 & 67.95 & 0.21 \\
\hline U65 & 61.64 & -0.60 \\
\hline
\end{tabular}

\subsection{Correlation analysis}

Pearson correlation analysis was performed among the morpho-physiological, and biochemical attributes of 12 short-day and 20 long-day onion genotypes under salt, drought, and combined salt+drought stress conditions (Fig. 2). The salt stress indicated positive correlation between chlorophyll contents and photosynthetic rate $(r=0.46)$, stomatal conductance rate $(r=0.45)$, transpiration rate $(r=0.66)$, bulb diameter $(r=0.58)$, and bulb weight $(r=0.64)$, whereas it showed negative correlation with malondialdehyde contents $(r=-0.47)$, proline contents $(r=-0.40)$, and leaf temperature $(r=-0.58)$. The bulb weight showed positive correlation with photosynthetic rate $(r=0.75)$, stomatal conductance $(r=0.76)$, transpiration rate $(r=0.90)$, number of leaves $(r=0.52)$, length of leaf $(r=0.76)$, bulb length $(r=0.80)$, and bulb diameter $(r=0.94)$, while it was negatively correlated with proline $(r=-0.39)$, malondialdehyde contents $(\mathrm{r}=-0.68)$ and leaf temperature ( $\mathrm{r}=-0.82)$ (Fig. 2a).

In response to drought stress a significant positive correlation was observed between photosynthesis and transpiration rate $(r=0.50)$, stomatal conductance $(r=0.65)$, length of leaf $(r=0.61)$, diameter of leaf $(r=0.59)$, bulb diameter $(r=0.79)$, bulb length $(r=0.50)$ and bulb weight $(r=0.76)$. The bulb weight showed strong positive correlation with photosynthetic rate $(r=0.77)$, stomatal conductance $(r=0.86)$, transpiration rate $(r=0.62)$, number of leaves $(r=0.52)$, length of leaf $(r=0.75)$, diameter of leaf $(r=0.76)$, bulb diameter $(r=0.94)$, and bulb length $(r=0.70)$, whereas it showed strong negative correlation with leaf temperature $(r=-0.92)$. The negative correlation was observed between malondialdehyde contents and photosynthetic rate $(r=-0.64)$, stomatal conductance $(r=-0.65)$, transpiration rate $(r=-0.64)$, number of leaves $(r=-0.36)$, length of leaf $(r=-0.67)$, diameter of leaf $(r=0.54)$, plant biomass $(r=-0.43)$, bulb diameter $(r=-0.69)$, bulb length $(r=-0.61)$, bulb weight ( $r=-0.73)$ (Fig. 2b).

The combined salt+drought stress showed positive correlation of photosynthetic rate with chlorophyll contents $(r=0.62)$, stomatal conductance $(r=0.74)$, transpiration rate $(r=0.64)$, bulb diameter $(r=0.81)$, bulb length ( $r=0.82)$, and bulb weight $(r=0.85)$, whereas it showed negative correlation with malondialdehyde contents $(r=-0.67)$, proline contents $(r=-0.64)$ and leaf temperature ( $r=-0.75)$ (Fig. 2c).

\subsection{Principal component analysis (PCA)}


Principal component analysis was performed for all the observed traits in response to three stress environments. The genotypes grouped into four different groups, blue ellipse shows tolerant group of genotypes, green ellipse grouped sensitive genotypes, red ellipse grouped moderate-tolerant genotypes, and black ellipse grouped moderate-sensitive genotypes (Fig. 2). The PCA biplot of the first two components (PC1 and PC2) for the salt stress explained 67.3\% variance for the tested traits in 32 onion genotypes (Fig. 2a). The PCA resulted in the distinctive separation of the genotypes based on the performance under salt stress conditions. The genotypes K18, K41, K51, K52, U12, U47, and U49 exhibited tolerance to the salt stress, as they showed higher gaseous exchange traits (photosynthetic rate, stomatal conductance, and transpiration rate), yield attributes (bulb weight, bulb diameter, and bulb length) also aggregated at near to the gaseous exchange traits that suggested their tolerance to salt stress. The genotypes K25, K28, K35, K58, U6, U17, U24, and U33 showed higher leaf temperature that accumulated higher malondialdehyde, and proline contents due oxidative burst forming a group of sensitive genotypes to salt stress (Fig. 2a).

The PCA biplot of the first two components (PC1 and PC2) for the drought stress explained $66.8 \%$ variance for the observed traits (Fig. 2b). The genotypes K35, K41, K52, K59, U12, U47, and U49 were tolerant to water deficit conditions as they showed better morphological growth with higher leaf length, number of leaves, and diameter of leaf, additionally they also showed higher gaseous exchange and yield traits. The genotypes K20, K25, U6, U10, U11, U17, and U31 were grouped as a sensitive genotype as they showed higher leaf temperature and differential accumulation of malondialdehyde and proline contents under drought stress conditions (Fig. 2b).

The PCA biplot of the first two components (PC1 and PC2) for the salt+drought stress explained $71.5 \%$ variance. The PCA exhibited the close association of different observed traits as shown by the loading byplots (Fig. 2c). The genotypes K35, K37, K39, K41, K52, U12, U47, and U49 formed a group of tolerant genotypes. Moreover, a smaller angle between different morpho-physiological attributes classified the genotypes to be tolerant. These genotypes were also plotted closer along the line direction of the vector. The genotypes K58, U6, U16, U17, U31, U33, and U31 were grouped as a sensitive genotype with the exposure to salt+drought stress conditions (Fig. 2c).

\subsection{Stability analysis}

AMMI biplot showed visual interpretation of interrelationship among 32 genotypes and three environments. Mean bulb weight is plotted against IPC1 (Interactive principal component) as shown in Fig. 3. The displacement along abscissa describes the additive (main) effects, while interactive effects can be explained by displacements along the ordinate. If a genotype or an environment has IPC1 score of nearly zero, it has small interaction effects and considered as stable. All three environments were diverse. Drought stress was observed to be the most stable environment as compared to SS and SD, since its IPC1 score is close to zero, indicating small interaction effects. Genotypes (U49, K41, U47, K52, U12) with high mean bulb weight combined with IPC1 score close to zero cluster on the right quadrat of AMMI biplot. These genotypes are considered as stable among all the environments. 


\subsection{Broad sense heritability}

The bulb weight is the most desired trait for abiotic stress breeding; therefore, it was considered to analyze the broad sense heritability. Genotypic variance and phenotypic variance were calculated to be 231.6 and 306.7 , respectively. Broad sense heritability was found to be 0.75 , which means that $75 \%$ variability in a trait was caused by genetic differences among genotypes. The trait was less influenced by the environment and allows the selection of genotypes for higher bulb weight (Table 3 ).

Table 3

AMMI ANOVA describing degree of freedom and mean squares of genotypes, environment and genotype by environment interactions, for bulb weight of 32 tested onion genotypes across three environmental conditions.

\begin{tabular}{|llllll|}
\hline Source & df & SS & MS & Probability \\
\hline Total & 287 & 163151.1 & 568.4708 & & \\
\hline TRT & 95 & 162810.4 & 1713.794 & 0 & $* \star *$ \\
\hline GEN & 31 & 85571.15 & 2760.36 & 0 & $* \star *$ \\
\hline ENV & 2 & 35332.82 & 17666.41 & 0 & $* \star *$ \\
\hline GxE & 62 & 41906.42 & 675.91 & 0 & $* \star *$ \\
\hline IPC1 & 32 & 26604.99 & 831.406 & 0 & $* \star *$ \\
\hline Residual & 30 & 15301.43 & 510.0476 & 0 & $* \star \star$ \\
\hline Error & 192 & 340.7285 & 1.77463 & & \\
\hline
\end{tabular}

\section{Discussion}

Abiotic stresses are the leading obstacles resulting in retarded growth, development and yield losses of onion bulb. Currently least information is available regarding the behavior of onion in response to salt or drought stress conditions (Chaudhry et al. 2020; Chaudhry et al. 2021; Gedam et al. 2021). However, according to our knowledge none of the study has observed the influence of combined salt+drought influence on onion morpho-physiological and biochemical attributes. Therefore, the current study was conducted for the screening of 32 short-day and long-day onion genotypes to varying stress conditions. The onion susceptibility to salt, drought, and combined salt+drought stress varied with the intensity of stresses. It was monitored that all the 32 onion genotypes manifested disruptions in observed traits with a grouping of sensitive and tolerant genotypes to stress environments.

All the 32 onion genotypes in this study acclimatized to salt, drought and combined salt+drought stresses showed contrasting behavior. The breeding lines K41, U47, U49 and commercial cultivar K52, showed higher gaseous exchange traits, in response to stress conditions, that suggested their inherent ability to maintain photosynthetic activity. In our earlier study we observed the similar behavior of tolerant onion 
genotypes (Chaudhry et al. 2020). The breeding lines K18, K20, K25, and commercial cultivar K51 showed lowest stomatal conductance, which means they were the most sensitive cultivars. Previous study indicated that with the fluctuation in environmental conditions the stomatal conductance decreases significantly (Sánchez-Virosta et al. 2021). Drought stress negatively influences photosynthesis activity and photosynthetic pigments, which results into decreased photosynthetic rate in plants. In salt and salt+drought stress, chlorophyll content (SPAD) was seen to be positively correlated with photosynthetic rate, stomatal conductance rate, transpiration rate. Higher chlorophyll content facilitates stable photosynthetic activity and enables plants to maintain their requirements under stress conditions, thereby in this study the breeding lines $\mathrm{K} 41, \mathrm{U} 12, \mathrm{U} 47, \mathrm{U} 49$, and commercial cultivar K52 showed better performance in contrast to other genotypes.

Decreased morphological traits are attributed to reduced cell expansion and cell growth which may led to decreased plant height under stress conditions. Similar changes in plant phenotype were observed in soybean crop during its vegetative and reproductive phase. Other studies also supported our results as drought stress and salt stress negatively affected the plant growth of onion (Hanci and Cebeci 2015). The breeding lines K25, U6, U17 and commercial cultivar K58 showed decline in morphological growth and yield traits, therefore grouped as sensitive genotypes. Results of this study are in accordance with Naghavi et al. (2013) that also reported decreased morphological growth functioning of maize. In a study related to leaf attributes of soybean under drought stress rendered that leaf traits were significantly decreased under water scarcity (Chowdhury et al. 2016; Barrios et al. 2005).

In our study, high variability in the response by different genotypes for leaf temp, proline and MDA was observed. The tolerant breeding lines K41, U12, U47, U49 and commercial cultivar K52 performed better under stress conditions as they accumulated least MDA and proline contents These results for regarding MDA and proline accumulation are in accordance with (Demirel et al. 2020).

In contrast, the breeding lines $\mathrm{K} 25, \mathrm{U} 6, \mathrm{U} 17$ and commercial cultivar K58 were designated as salt sensitive cultivars as they exhibited higher temperature, MDA and proline and poor yield under stress. In our earlier study we observed the similar results where a disruption in membrane stability due to MDA accumulation (Chaudhry et al. 2021). Since proline is key player in plant tolerance that accumulates to alleviate oxidative stress in plant tissues. Higher proline accumulation in these genotypes suggested that they were under higher influence of oxidative stress.

The bulb characteristics are the most desired attributes for screening of onion genotypes. Differential response of onion genotypes was observed in response to stress conditions. The breeding lines K18, K41, $\mathrm{U} 12, \mathrm{U} 47, \mathrm{U} 49$ and commercial cultivars K51, K52, exhibited tolerance to the salt stress, as they showed higher yield attributes (bulb weight, bulb diameter, and bulb length), whereas the breeding lines K25, K28, $\mathrm{K} 35, \mathrm{U6}, \mathrm{U17}, \mathrm{U} 24, \mathrm{U} 33$ and commercial cultivar K58 showed sensitivity to salt stress. The imposition of drought stress revealed that the breeding lines K35, K41, U12, U47, U49 and K52, K59 commercial cultivars were tolerant to water deficit conditions, while the breeding lines $\mathrm{K} 20, \mathrm{~K} 25, \mathrm{U} 6, \mathrm{U} 10, \mathrm{U} 11, \mathrm{U} 17$, and U31 were grouped as a sensitive genotype. The exposure of genotypes to combined salt+drought 
stress exhibited that the breeding lines K35, K37, K39, K41, U12, U47, U49 and commercial cultivar K52, were tolerant, whereas the breeding lines $\mathrm{U} 6, \mathrm{U} 16, \mathrm{U} 17, \mathrm{U} 31, \mathrm{U} 33$, and commercial cultivar K58, were grouped as a sensitive genotype. Results regarding reduction in onion bulb traits are in accordance with our earlier finding (Chaudhry et al. 2020). Additionally, a recent study conducted by Gedam et al. (2021) also screened the onion genotypes based on yield reduction.

In this study the reduction in bulb characteristics is attributed to decrease in gaseous exchange traits with damage to chlorophyll contents, that resulted in oxidative burst. As sensitive genotypes excelled in accumulating malondialdehyde and proline contents that suggested these genotypes were under higher influence of the applied stresses. The results of earlier studies showed a reduction in onion bulb yield due to restrained uptake of nutrients, likely reduction in photosynthetic rate with the closure of stomata that supports the results of this study [Chaudhry et al. 2020; Zheng et al. 2013; Wakchaure et al. 2018).

Correlation analysis further strengthened that tested traits resulted in higher bulb weight of the onion. As all the morpho-physiological traits except leaf temperature were significantly positive correlated with the bulb weight. Contrarily biochemical traits showed strong negative correlation under stress regimes (Fig. 1). Therefore, the genotypes that exhibited improved gaseous exchange traits, less damage to chlorophyll contents, a greater number of leaves and least stunted growth were tolerant. Contrarily the genotypes that showed higher membrane damage (MDA) and greater proline contents were sensitive with weak tolerance mechanism to stress conditions. Our results are in accordance with earlier report in potato (Demirel et al. 2020).

The PCA analysis revealed the grouping the of the onion genotypes as tolerant, sensitive, moderatetolerant and moderate-sensitive (Fig. 2). The biplot clearly grouped the onion genotypes into tolerant and sensitive genotypes based on their performance in response to salt, drought and salt+drought stress conditions. The morpho-physiological traits accumulated in positive quadrat and close to each other that supported that tolerance behavior was due to their overall performance of these genotypes to each stress conditions, whereas proline, MDA and leaf temperature was in negative quadrat that resulted in oxidative stress in sensitive genotypes.

A biplot created between PC1 and PC2 showed a clear pattern of grouping genotypes along the vector line. The outstanding performance of genotypes for a specific trait was plotted closer to the vector line. Genotypes and traits that lie far away from the origin have better breeding potential than the other genotypes. Current study classified the breeding lines K25, U6, U17 and commercial cultivar K58 as sensitive to all stress conditions. Contrarily the breeding lines U12, U47, U49 and commercial cultivar K52 exhibited tolerance mechanism and classified as tolerant to all stress conditions. Similar findings were reported by (Gedam et al. 2021). Our results regarding PCA analysis was further corroborated by results reported by (Al-Ashkar et al. 2019). Furthermore, it was also reported by (Grzesiak et al. 2019) that tolerant genotypes were plotted in the positive quadrat near to stress promising traits.

AMMI stability analysis validated that all three stress environments were diverse and suggested differential genotypes response to the exposed stress environments (Naeem and Caliskan 2020). The 
onion breeding lines $\mathrm{K} 41, \mathrm{U} 12, \mathrm{U} 47, \mathrm{U} 49$ and commercial cultivar K52 clustered in the right quadrat were considered as stable genotypes to applied stress conditions. These genotypes had desired traits that can be used by the breeders for breeding of high yielding onion varieties under varying environments. Our results are comparable to findings of (Edabi et al. 2019).

In our study the significant differences among tested onion genotypes indicated the presence of genetic variation for the measured bulb weight trait. The improvement in onion towards stress tolerance depends on genetic variability for determination of stress tolerance. We quantified heritability that provided information about the magnitude of genetic and environmental variation (Roy and Shil 2020). It is also potent tool to predict phenotypic reliability that contributes for breeding values. We observed higher heritability for bulb weight that suggested that it was due to genetic difference rather than the influence of environment on genotypes. Thereby it can be exploited as consistent criteria for screening of different genotypes to abiotic stress tolerance.

\section{Conclusion}

Current study explored the potential of 32 onion breeding lines and commercial cultivars to contrasting stress conditions. The genotypes were screened based on their morpho-physiological and biochemical changes. Traits analyzed in this study are the major selection criterion for the screening of stress tolerant and susceptible cultivars and can be used for the screening of large onion germplasm. The breeding lines K41, U47, U49 and commercial cultivar K52, were found to be stable across different stress conditions. These genotypes showed desirable traits to be used as a breeding material for abiotic stress breeding.

\section{Declarations}

\section{Funding}

This study was funded by TÜBITAK-KAMAG with a project number of 117G023 (Kışlık Sebze Islahında Hat ve/veya Çeşit Geliştirme).

\section{Conflict of interest}

The authors declare no conflict of interest.

\section{Authors contributions}

ZNÖG and AFG conceptualized the idea and designed the study. UKC and MDJ performed the experiment, collected the data, analyzed the data and wrote the initial draft. ZNÖG and AFG edited and reviewed the draft of paper. All the Authors, read and approved the final draft.

\section{References}


Al-Ashkar I, Alderfasi A, El-Hendawy S, Al-Suhaibani N, El-Kafafi S, Seleiman MF (2019) Detecting salt tolerance in doubled haploid wheat lines. Agronomy 9: 211. https://doi.org/10.3390/agronomy9040211

Asim A, Gökçe ZNÖ, Bakhsh A, Çayli IT, Aksoy E, Çalişkan S, Çalişkan ME, Demirel U (2021) Individual and combined effect of drought and heat stresses in contrasting potato cultivars overexpressing miR172b-3p. Turk J Agric For 45: 651-668. https://doi:10.3906/tar-2103-60

Barrios A, Hoogenboom G, Nesmith DS (2005) Drought stress and the distribution of vegetative and reproductive traits of a bean cultivar. Sci Agri 62: 18-22.

Brewster JL (1994) March. Environmental physiology of the onion: towards quantitative models for the effects of photoperiod, temperature and irradiance on bulbing, flowering and growth. In I International Symposium on Edible Alliaceae 433 (pp. 347-374). https://10.17660/ActaHortic.1997.433.37

Chaudhry UK, Gökçe ZN, Gökçe AF (2020) Effects of Salinity and Drought Stresses on the PhysioMorphological Attributes of Onion Cultivars at Bulbification Stage. Int J Agric Biol 24(6): 168189. https://doi:10.17957/IJAB/15.1611

Chaudhry UK, Gökçe ZNÖ, Gökçe AF (2021) Drought and salt stress effects on biochemical changes and gene expression of photosystem II and catalase genes in selected onion cultivars. Biologia 115. https://doi.org/10.1007/s11756-021-00827-5

Chaudhry UK, Junaid MD, Gökçe AF (2021) Influence of Environmental Adversities on Physiological Changes in Plants. In Developing Climate-Resilient Crops, CRC Press, pp. 85-

110. https://doi.org/10.1201/9781003109037

Chowdhury JA, Karim MA, Khaliq QA, Ahmed AU, Khan MSA (2016) Effect of drought stress on gas exchange characteristics of four soybean genotypes. Bangladesh J Agr Res 41: 195-205. doi: https://doi.org/10.3329/bjar.v41i2.28215

Clay DE, Clay SA, Reitsma KD, Dunn BH, Smart AJ, Carlson GG, Horvath D, Stone JJ (2014) Does the conversion of grasslands to row crop production in semi-arid areas threaten global food supplies? Glob Food Sec 3: 22-30. https://doi.org/10.1016/j.gfs.2013.12.002

Demirel U, Morris WL, Ducreux LJ, Yavuz C, Asim A, Tindas I, Campbell R, Morris JA, Verrall SR, Hedley PE, Gokce ZN (2020) Physiological, biochemical, and transcriptional responses to single and combined abiotic stress in stress-tolerant and stress-sensitive potato genotypes. Front Plant Sci 11: 169. https://doi.org/10.3389/fpls.2020.00169

Drinkwater WO, Janes, BE (1957) Relation of potential evapotranspiration to environment and kind of plant. Eos, Transactions American Geophysical Union, 38: 524-528.

Ebadi AA, Hallajian MT, Kordrostami M (2019) The genetic variation and stability analysis of rice mutant lines using AMMI model under normal and drought stress conditions. Genetika 51: 687- 
FAOSTAT. 2019. Food and Agriculture Organization of the United Nations. Rome: FAO.

Gedam PA, Thangasamy A, Shirsat DV, Ghosh S, Bhagat KP, Sogam OA, Gupta AJ, Mahajan V, Soumia PS, Salunkhe VN, Khade YP. 2021. Screening of Onion (Allium cepa L.) Genotypes for Drought Tolerance Using Physiological and Yield Based Indices Through Multivariate Analysis. Front Plant Sci 12:

122. https://doi.org/10.3389/fpls.2021.600371

Ghodke P, Khandagale K, Thangasamy A, Kulkarni A, Narwade N, Shirsat D, Randive P, Roylawar P, Singh I, Gawande SJ, Mahajan V (2020) Comparative transcriptome analyses in contrasting onion (Allium cepa L.) genotypes for drought stress. PloS one, 15: 237457. https://doi.org/10.1371/journal.pone.0237457

Gökçe AF (2018) Classical Genetics on Gene Mapping. The Allium Genomes, pp.5366. https://doi.org/10.1007/978-3-319-95825-5_4

Golldack D, Li C, Mohan H, Probst N (2014) Tolerance to drought and salt stress in plants: unraveling the signaling networks. Front Plant Sci 5: 151. https://doi.org/10.3389/fpls.2014.00151

Grzesiak S, Hordyńska N, Szczyrek P, Grzesiak MT, Noga A, Szechyńska-Hebda M. 2019. Variation among wheat (Triticum easativum L.) genotypes in response to the drought stress: I-selection approaches. J Plant Interact 14: 30-44. https://doi.org/10.1080/17429145.2018.1550817

Gürel F, Öztürk NZ, Uçarlı C (2016) Transcriptomic responses of barley (Hordeum vulgare L.) to drought and salinity. In Plant Omics: Trends and Applications (pp. 159-188). Springer,

Cham. https://doi.org/10.1007/978-3-319-31703-8_

Hanci F, Cebeci E (2015) Comparison of salinity and drought stress effects on some morphological and physiological parameters in onion (Allium cepa L.) during early growth phase. Bulga J Agric Sci 21:1204-1210.

Havey MJ, Ghavami F (2018) Informativeness of single nucleotide polymorphisms and relationships among onion populations from important world production regions. J Am Soc Hortic Sci 143: $34-$ 44. https://doi.org/10.21273/JASHS04277-17

Heath RL, Packer L (1968) Photoperoxidation in isolated chloroplasts: I. Kinetics and stoichiometry of fatty acid peroxidation. Archives of biochemistry and biophysics, 125(1), pp.189198. https://doi.org/10.1016/0003-9861(68)90654-1

Junaid MD, Chaudhry UK. Gökçe AF. 2021. Climate Change and Plant Growth-South Asian Perspective. Climate Change and Plants: Biodiversity, Growth and Interactions, p.37. https://doi.org/10.1201/9781003109037 
Kinraide TB (1999) Interactions among $\mathrm{Ca} 2+, \mathrm{Na}+$ and $\mathrm{K}+$ in salinity toxicity: quantitative resolution of multiple toxic and ameliorative effects. J Exp Bot 50: 1495-

1505. https://doi.org/10.1093/jxb/50.338.1495

Mubarak I, Hamdan A (2018) Onion crop response to regulated deficit irrigation under mulching in dry Mediterranean region. J Hortic Res 26(1).

Naeem M, Caliskan ME (2020) Comparison of methods for dry matter content determination in potato using multi-environments field data and stability statistics. Turkish J Field Crop 25: 197207. https://doi.org/10.17557/tjfc.742244

Naghavi MR, Aboughadareh AP, Khalili M (2013) Evaluation of drought tolerance indices for screening some of corn (Zea mays L.) cultivars under environmental conditions. Notulae Scientia Biologicae, 5(3): 388-393. https://doi.org/10.15835/nsb539049

Rao NS, Shivashankara KS, Laxman RH (2016) Abiotic stress physiology of horticultural crops (Vol. 311). India: Springer. https://doi.org/10.1007/978-81-322-2725-0

Roy SC, Shil P (2020) Assessment of genetic heritability in rice breeding lines based on morphological traits and caryopsis ultrastructure. Sci Rep 10: 1-17. https://doi.org/10.1038/s41598-020-63976-8

Sánchez-Virosta Á, Sadras VO, Sánchez-Gómez D (2021) Phenotypic plasticity in relation to inter-cultivar variation of garlic (Allium sativum L.) functional performance and yield-stability in response to water availability. Sci Hortic 285:110-128. https://doi.org/10.1016/j.scienta.2021.110128

Shahzad S, Khan MY, Zahir ZA, Asghar HN, Chaudhry UK, 2017. Comparative effectiveness of different carriers to improve the efficacy of bacterial consortium for enhancing wheat production under salt affected field conditions. Pakistan Journal of Botany, 49: 1523-1530.

Singh VK, Singh AK, Singh PP, Kumar A (2018) Interaction of plant growth promoting bacteria with tomato under abiotic stress: a review. Agriculture, Ecosystems \& Environment, 267: 129140. https://doi.org/10.1016/j.agee.2018.08.020

Steel RGD, JH Torrie, DA Dicky (1997) Principles and Procedures of Statistics-A Biomedical Approach. McGraw-Hill International Co., Singapore

Toker C (2004) Estimates of broad-sense heritability for seed yield and yield criteria in faba bean (Vicia faba L.)". Hereditas, 140: 222-225. https://doi.org/10.1111/j.1601-5223.2004.01780.x

Wakchaure GC, Minhas PS, Meena KK, Singh NP, Hegade PM, Sorty AM (2018) Growth, bulb yield, water productivity and quality of onion (Allium cepa L.) as affected by deficit irrigation regimes and exogenous application of plant bio-regulators. Agric Water Manag 199: 110. https://doi.org/10.1016/j.agwat.2017.11.026 
Zheng J, Huang G, Wang J, Huang Q, Pereira LS, Xu X, Liu H (2013) Effects of water deficits on growth, yield and water productivity of drip-irrigated onion (Allium cepa L.) in an arid region of Northwest China. Irrigation Science, 31: 995-1008. http://doi.org/10.1007/s00271-012-0378-5

\section{Figures}

A

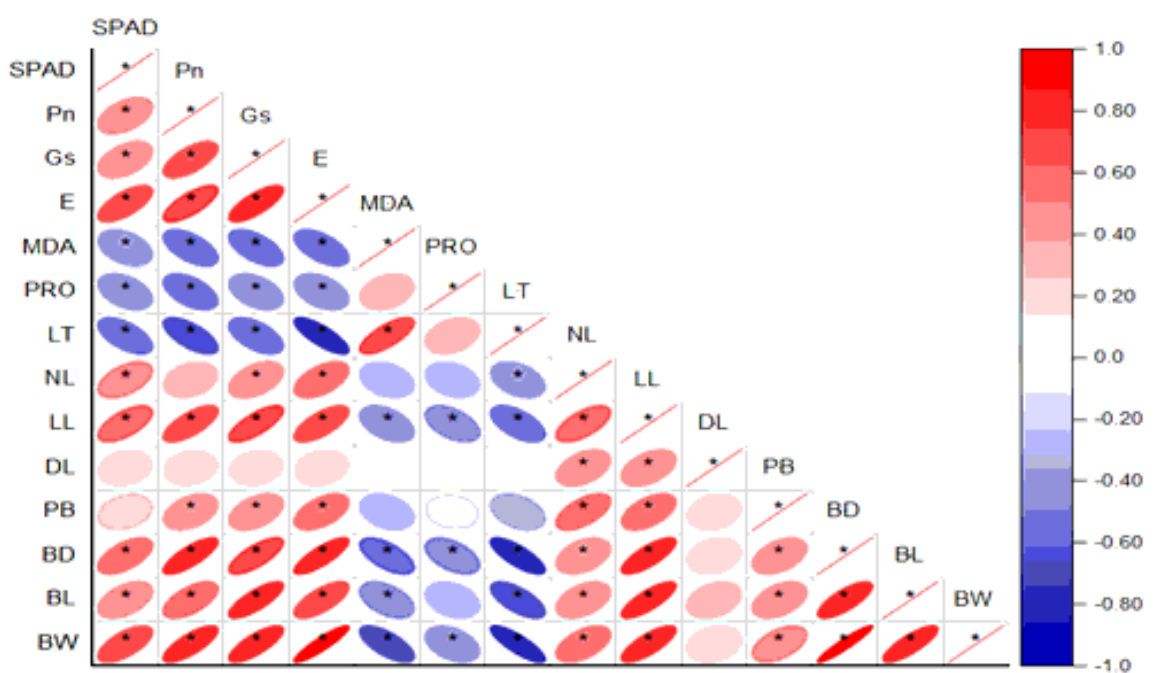

B

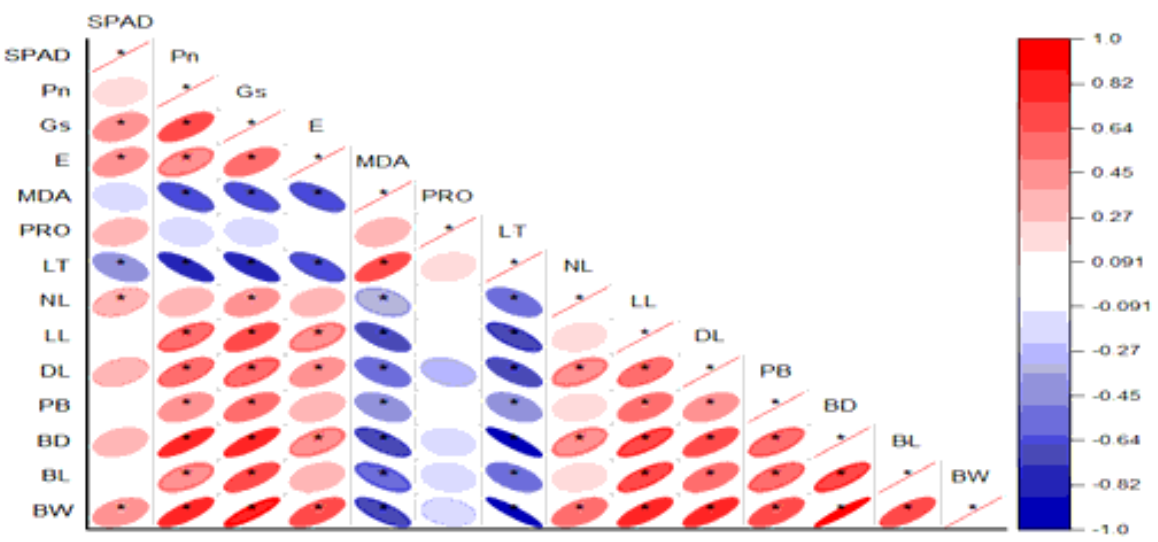

C

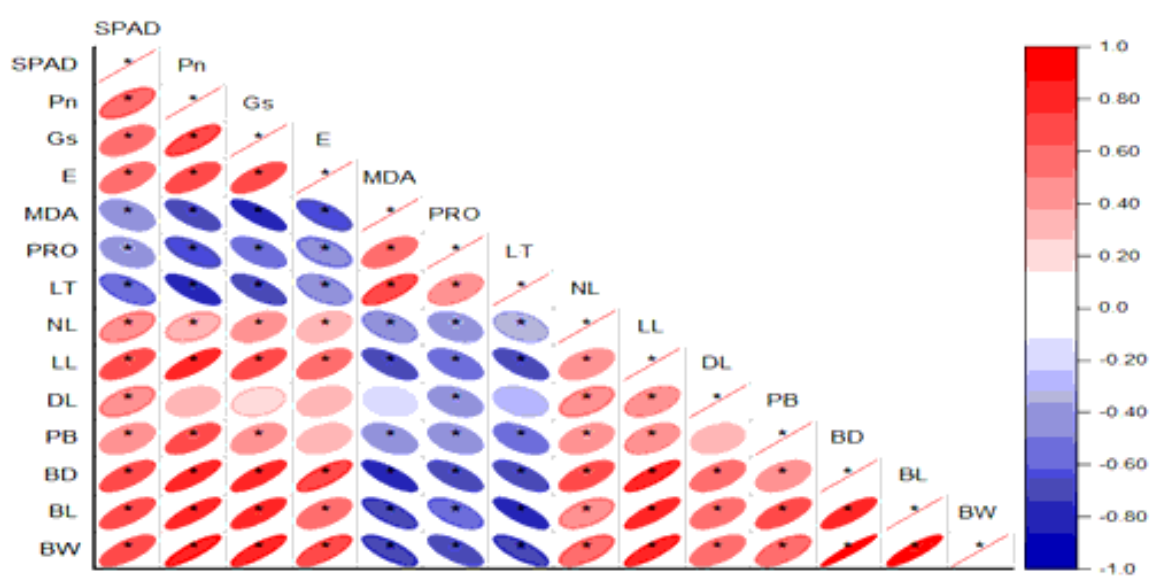


Pearson correlation for different attributes observed in response to salt stress (a), drought stress (b) and salt+drought stress in 32 onion genotypes, *significant at $p \leq 0.05$, SPAD chlorophyll content, Pn Photosynthetic rate, g stomatal conductance, E transpiration rate, MDA malondialdehyde contents, PRO proline contents, LT leaf temperature, NL number of leaves, LL leaf length, DL leaf diameter, PB plant biomass, BD bulb diameter, BL bulb length, BW bulb weight

A

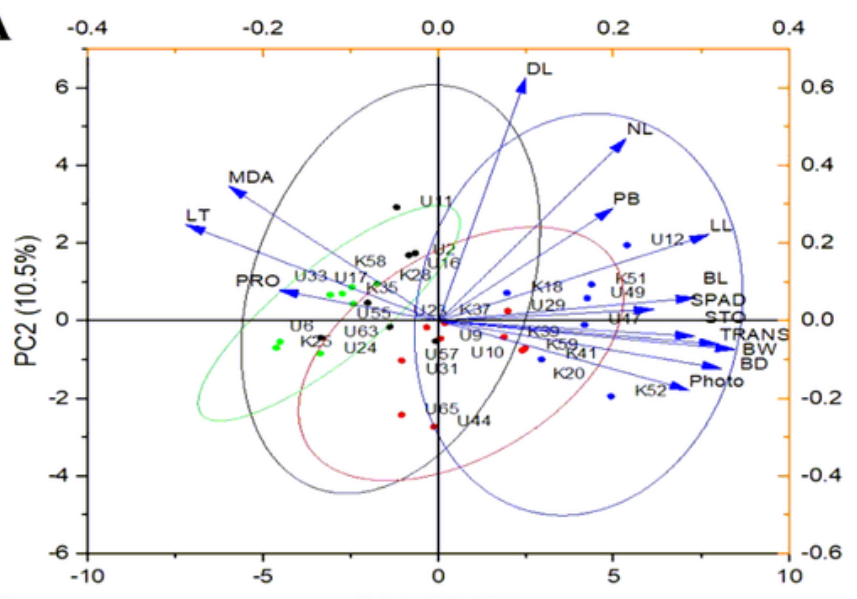

B
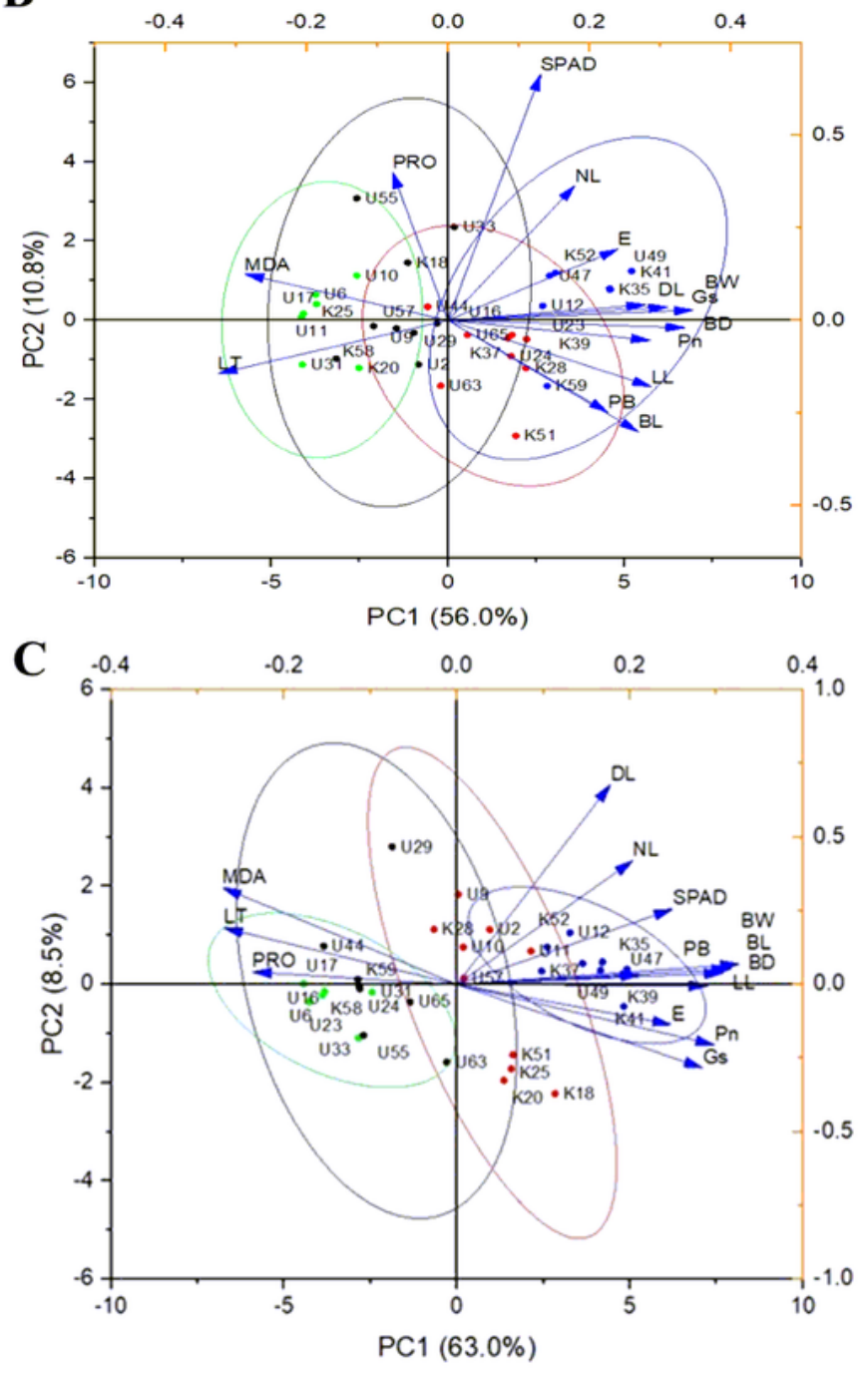

.5

.5

0.5

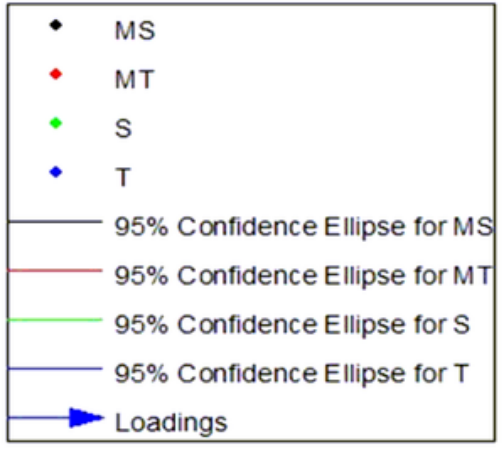

Figure 2

Page 18/19 
Principal component analysis of 32 onion genotypes to salt stress (a), drought stress (b) and salt+drought stress (c), SPAD chlorophyll content, Pn Photosynthetic rate, g stomatal conductance, E transpiration rate, MDA malondialdehyde contents, PRO proline contents, LT leaf temperature, NL number of leaves, LL leaf length, DL leaf diameter, PB plant biomass, BD bulb diameter, BL bulb length, BW bulb weight

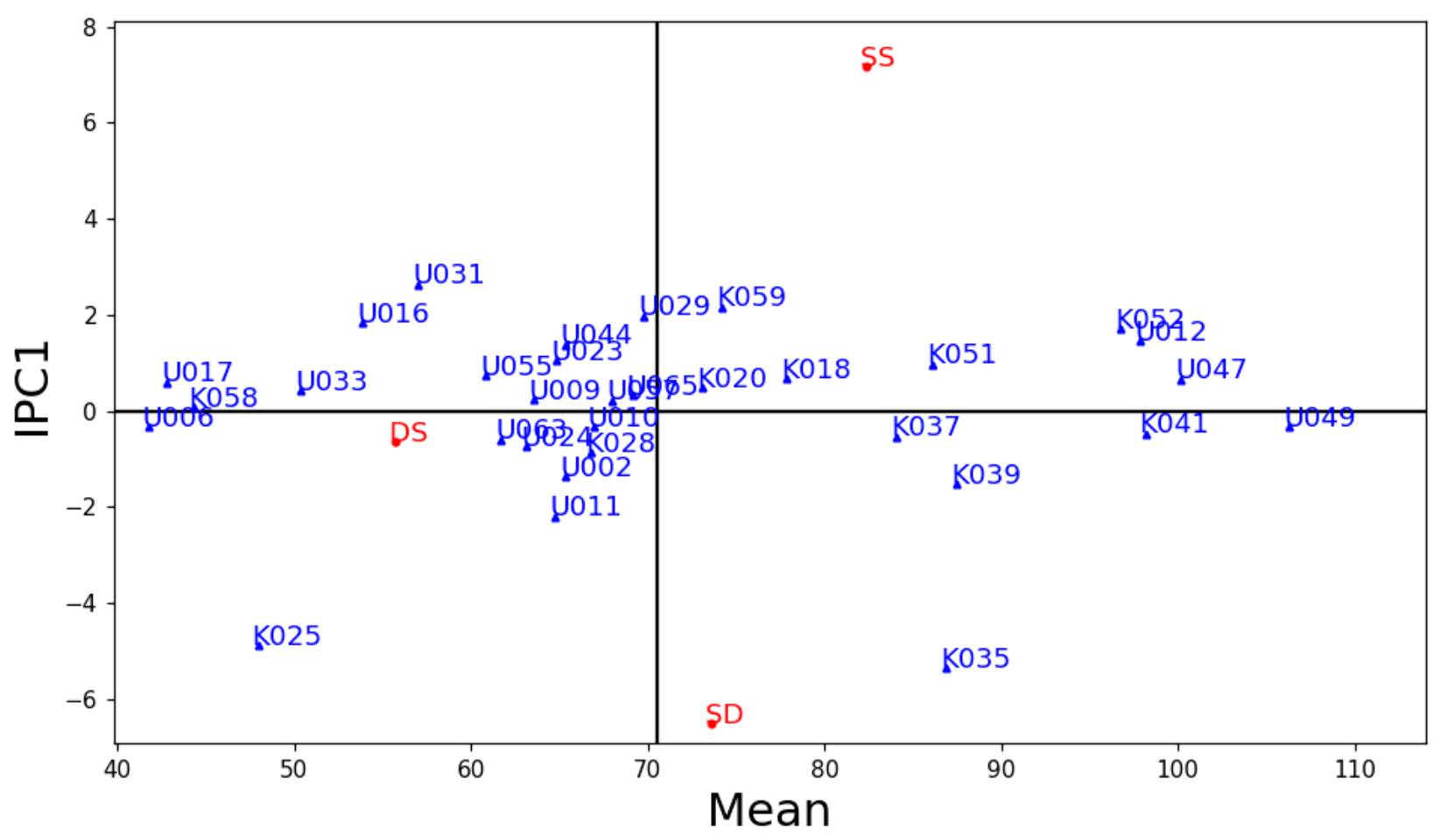

Figure 3

AMMI biplot for mean bulb weight of 32 onion genotypes (blue dots) and three environments (SS salt stress, DS drought stress, and SD salt+drought stress) plotted against IPC1 (Interactive principal component)

\section{Supplementary Files}

This is a list of supplementary files associated with this preprint. Click to download.

- SupplementaryFile.docx 